\title{
Desafios no Ensino a Distância: Soluções Computacionais para a Aprendizagem Colaborativa com Computação Afetiva
}

\author{
Leonam C. Oliveira ${ }^{1}$, Carolina M. Oliveira ${ }^{1}$, Leander C. Oliveira ${ }^{2}$, Andrey R. \\ Pimentel $^{1}$
}

${ }^{1}$ Departamento de Informática - Universidade Federal do Paraná (UFPR) Curitiba - PR - Brasil

${ }^{2}$ Programa de pós-graduação em Tecnologia e Sociedade - Universidade Tecnológica Federal do Paraná (UTFPR) - Curitiba - PR - Brasil

\{leonamcdeoliveira, carolmoliveiraa, leanderdeoliveira\}gmail.com, andreydinf.ufpr.br

Abstract. Affective computing has aided collaborative learning in the presential educational context, and it is often necessary to use computational resources that may not be available in e-learning education. Taking into account the constant increase in enrollment in this modality, it is important to propose computational solutions in which data collection instruments are also available for e-learning.

Resumo. A computação afetiva tem auxiliado a aprendizagem colaborativa no contexto educacional presencial, sendo muitas vezes necessário a utilização de recursos computacionais que podem não estar disponíveis no ensino a distância. Levando em conta o aumento constante de matrículas nesta modalidade, é importante propor soluções computacionais em que sejam selecionados instrumentos de coleta de dados que estejam disponíveis também para o ensino a distância.

\section{Desafios da computação afetiva no ensino a distância}

Compreender a computação afetiva e a sua aplicação em ferramentas educacionais é um desafio, segundo Landowska (2013). A computação afetiva é uma área de pesquisa recente, sendo definida como a computação que se relaciona, surge ou se influencia de emoções e outros fenômenos afetivos [Pudane et al., 2018]. Estudos recentes corroboram com a necessidade de pesquisas na aprendizagem colaborativa que envolvam a computação afetiva, conforme apontam Reis et al. (2018).

A pesquisa de Eligio et al. (2012), cita a detecção de emoção e personalidade como benefícios para a aprendizagem colaborativa. Alguns desafios de pesquisa na área são propostos por Reis et al. (2018), sendo exemplos: envolver a consciência emocional, a formação de grupos e a interação entre estudantes levando em conta aspectos afetivos que tenham influência no processo educativo. Grande parte das soluções computacionais são voltadas ao ensino presencial, porém, quando considerado o Ensino a Distância (EAD), os aspectos afetivos são ainda mais importantes e podem ser fatores cruciais para uma educação bem-sucedida [Landowska, 2013]. 
VIII Congresso Brasileiro de Informática na Educação (CBIE 2019)

Anais do VIII Workshop de Desafios da Computação aplicada à Educação (DesafIE 2019)

Conforme o Censo da Educação Superior [INEP, 2017], houve um crescimento de matrículas em cursos de graduação EAD no Brasil. Enquanto em 2007 o EAD correspondia a $15,4 \%$ das matrículas, hoje passou a corresponder a 1/3 das matrículas no ensino superior. $\mathrm{Na} \mathrm{EAD}$ as tecnologias digitais são recursos importantes para oferecer interatividade, colaboração e construção do conhecimento [Zanelato, 2009]. Com a popularização do EAD, há resultados eficazes e eficientes quando há a possibilidade de reconhecer as emoções dos estudantes [Lin et al., 2016].

Dentre os algoritmos desenvolvidos para detectar os estados afetivos estão os baseados em processamento de informações visuais, análise de movimentação do corpo, análise de textos, processamento de sinais de voz, interpretação de mensurações fisiológicas, entre outros, conforme cita Landowska (2013). Porém, é necessário adequar as estratégias levando em conta a disponibilidade a partir do ambiente ao qual pretende-se aplicar a computação afetiva [Landowska, 2013].

Segundo Landowska (2013), para detectar os estados afetivos, é importante analisar os instrumentos disponíveis, normalmente dispositivos periféricos, como mouse e teclado, entradas de texto e algumas vezes câmeras e microfones, que nem sempre estão disponíveis. Ainda, é necessário que estes canais de entrada, para detectar estados afetivos, sejam combinados para melhorar a acurácia do reconhecimento. Considera-se também que os canais de entrada podem não ser os mais confiáveis, sendo um dos desafios o de estimar a qualidade destas detecções no contexto de uso.

Diante do exposto, tem-se como desafio desenvolver soluções computacionais com qualidade para utilização no EAD. É preciso, inicialmente, levar em conta que há a possibilidade da não disponibilidade de recursos como câmeras, microfones, dentre outros. Há necessidade de adaptação dos instrumentos de coleta das informações para a detecção de estados afetivos, sendo estes escolhidos com base na disponibilidade de cada estudante no seu local de estudo.

Cada estudante pode utilizar um dispositivo diferente para acesso às suas disciplinas, como notebooks, PCs, tablets e smartphones, por exemplo. Desse modo, é necessário esforços visando compreender as possibilidades de aplicação da computação afetiva no ensino a distância. Tem-se como desafio o de detectar estados afetivos, como emoção e/ou personalidade, de estudantes que não tem a disponibilidade dos mesmos instrumentos utilizados para coleta de dados no ensino presencial, sem que a qualidade das tecnologias computacionais seja perdida.

São necessários estudos que comparem as tecnologias computacionais desenvolvidas para a educação presencial e para a EAD no contexto específico apresentado neste estudo. Compreender os resultados da detecção de estados afetivos aplicados a aprendizagem colaborativa no ensino presencial podem ajudar a entender os instrumentos de coleta que poderiam ser usados na EAD, levando em conta as peculiaridades ao aplicar a computação afetiva neste contexto.

\section{Agradecimentos}

O presente trabalho foi realizado com apoio da Coordenação de Aperfeiçoamento de Pessoal de Nível Superior - Brasil (CAPES) - Código de Financiamento 001. 


\section{Referências}

Eligio, U. X., Ainsworth, S. E. e Crook, C. K. (2012). Emotion understanding and performance during computer-supported collaboration. Computers in Human Behavior, 28(6), 2046-2054.

INEP (2017). Censo da Educação Superior: Notas Estatísticas 2017. Ministério da Educação. Acessado em 26/08/2019. Disponível em: http://download.inep.gov.br/educacao_superior/censo_superior/documentos/2018/ce nso_da_educacao_superior_2017-notas_estatisticas2.pdf

Landowska, A. (2013). Affective computing and affective learning-methods, tools and prospects. Stara strona magazynu EduAkcja, 5(1).

Lin, H. C. K., Su, S. H., Chao, C. J., Hsieh, C. Y. e Tsai, S. C. (2016). Construction of multi-mode affective learning system: taking affective design as an example. Journal of Educational Technology \& Society, 19(2), 132-147.

Pudane, M., Petrovica, S., Lavendelis, E. e Anohina-Naumeca, A. (2018). Challenges in the Development of Affective Collaborative Learning Environment with Artificial Peers. Applied Computer Systems, 23(2), 101-108.

Reis, R. C. D., Isotani, S., Rodriguez, C. L., Lyra, K. T., Jaques, P. A. e Bittencourt, I. I. (2018). Affective states in computer-supported collaborative learning: Studying the past to drive the future. Computers \& Education, 120, 29-50.

Zanelato, A. P. A. (2009). A avaliação no ensino a distância. ETIC-Encontro de Iniciação Científica. ISSN 21-76-8498, 5(5). 